\title{
The role of a prone setup in breast radiation therapy
}

\author{
Nelly Huppert, Gabor Jozsef, Keith DeWyngaert and Silvia Chiara Formenti * \\ Department of Radiation Oncology, New York University School of Medicine, New York University Langone Medical Center, New York, NY, USA
}

\section{Edited by:}

Anatoly Dritschilo, Georgetown

University School of Medicine, USA

Reviewed by:

Brian Timothy Collins, Georgetown Hospital, USA

Nicole Sunderland Anderson, Brown University School of Medicine, USA

Arnold Malcolm, Vanderbilt University

Medical Center, USA

*Correspondence:

Silvia Chiara Formenti, New York

University Clinical Cancer Center, 160

East 34th Street, Room 123, New

York, NY 10016, USA.

e-mail: silvia.formenti@nyumc.org
Most patients undergoing breast conservation therapy receive radiotherapy in the supine position. Historically, prone breast irradiation has been advocated for women with large pendulous breasts in order to decrease acute and late toxicities. With the advent of CT planning, the prone technique has become both feasible and reproducible. It was shown to be advantageous not only for women with larger breasts but in most patients since it consistently reduces, if not eliminates, the inclusion of heart and lung within the field. The prone setup has been accepted as the best localizing position for both MRI and stereotactic biopsy, but its adoption has been delayed in radiotherapy. New technological advances including image-modulated radiation therapy and image-guided radiation therapy have made possible the exploration of accelerated fractionation schemes with a concomitant boost to the tumor bed in the prone position, along with better imaging and verification of reproducibility of patient setup. This review describes some of the available techniques for prone breast radiotherapy and the available experience in their application. The NYU prone breast radiotherapy approach is discussed, including a summary of the results from several prospective trials.

Keywords: breast cancer, prone setup

\section{INTRODUCTION}

As an alternative to mastectomy for breast cancer, approaches that permitted breast conservation began in France in the thirties and from the very beginning, included radiation therapy (Baclesse et al., 1939). Nearly half a century later, breast conservation surgery with radiotherapy proved to achieve the same outcome as mastectomy, for both local control and survival (Fisher et al., 2002; Veronesi et al., 2002).

During this phase, the radiation technique used identified the breast as the target based on clinical references and anatomical boundaries which were used to reproduce a daily setup. Initially this setup was only clinical, but in the sixties radiation simulator machines were introduced, enabling a more accurate daily positioning based on fluoroscopic and radiological references to bony landmarks obtained before initiation of treatment, in a preparation session defined as simulation. At simulation, reference images are generated, to be compared to the verification images obtained directly from the treatment machine, documenting the beam's eye view, every few radiotherapy sessions. If differences are found the necessary adjustments are introduced. The advent of radiotherapy planning based on CT imaging in the eighties (Jelden et al., 1976) allowed the possibility of obtaining body contours as well as anatomical visualization of thoracic organs, thus revolutionizing the accuracy of treatment planning. In 1991, threedimensional treatment planning for breast cancer was introduced (Solin et al., 1991) and established as routine almost a decade later.

From the beginning it was noted that patients with large breast size experience increased acute and late skin toxicities, particularly at the infra-mammary fold. While the option of breast conservation therapy (BCT) was also offered to them, cosmetic results were found to be inferior (Gray et al., 1991) and for this reason some cautioned against the use of radiotherapy in this group (Bentel et al., 1999). Late effects of increased fibrosis, retraction, and telengiectasias were frequently noted. This was likely attributed to the increased separation distance of the target volume and frequent dose inhomogeneity due to the shape of the breast tissue that often will flop over laterally, when supine.

\section{A PRONE SETUP FOR WOMEN WITH LARGE BREAST SIZE}

Several techniques have been explored to correct for these technical limitations in an attempt to assure a good cosmetic outcome for women with large breast size who necessitate radiation therapy.

Attempts to improve the homogeneity of dose distribution included: (a) changing/mixing beam energies (Monson et al., 1997), (b) introducing immobilization devices that displace the breast from the chest wall (Zierhut et al., 1994), and (c) modifying patient positioning, for instance with the lateral decubitus (LD) position (Baclesse et al., 1939; Cross et al., 1989). Approaches which included the construction of a thermoplastic mold or utilized a reinforced PVC ring and Styrofoam ${ }^{\mathrm{TM}}$ (Bentel and Marks, 1994; Zierhut et al., 1994) to pull the lateral breast tissue anteriorly and upright when supine and to decrease the separation of the tangents, as well as reducing the contact of the breast tissue with the chest wall at the infra-mammary fold were proposed. These devices failed to gain wide acceptance and standard application likely due to decreased reproducibility and patient discomfort.

Since the inception of this area of research, investigators have explored the potentially beneficial dosimetric effects of treating patients in the LD position. The LD position was introduced by Baclesse in the 1950s and has been applied to thousands of breast 
cancer patients treated at the Institute Curie and at other radiotherapy centers in France (Fourquet et al., 1991). The greatest advantage of the LD technique is in decreasing the separation by reducing breast thickness, thus decreasing inhomogeneity and the need for beam modifiers. The greatest challenge is the difficulty in reproducing the daily setup as well as in obtaining an accurate contour for calculation of dose distribution before CT simulation was available to verify this setup. The technique was originally designed to treat patients with a fixed gantry but it was maintained after the introduction of rotating gantries to treat women with larger breasts, instead of treating them in supine position.

\section{THE MSKCC EXPERIENCE OF PRONE RADIOTHERAPY}

In 1994, investigators from Memorial Sloan-Kettering Cancer Center (MSKCC) reported prone breast radiotherapy as a viable alternative to the classic supine positioning for patients with large medial to lateral separation (Merchant and Mccormick, 1994). A platform for prone breast radiotherapy was developed to add to the treatment couch that was made commercially available (Victoreen $^{\circledR}$, Model 37-018; Figure 1). The platform has a modifiable aperture (by sliding an insert in the lateral direction) through which the index breast hangs away from the thorax by gravity when the patient is prone. The ipsilateral arm is placed either at the side or above the head. A wedge under the contralateral side achieves an axial rotation of the patient toward the treated breast. The medial and lateral borders of the breast tissue are clinically assessed and define the field for two tangential, parallel opposed photon beams.

In an initial report, representative isodose distributions were generated for a patient simulated in both prone and supine positions. The isodose distribution was done in the transverse plane for both techniques and optimized with the use of beam wedges. More homogeneous isodose distributions were found for the patients in the prone position. The technique, as initially proposed by the MSKCC group, did not require CT simulation (Merchant and Mccormick, 1994). In a subsequent report of 245 patients treated from 1992 to 2004, tolerability and efficacy of this approach were confirmed (Grann et al., 2000; Stegman et al., 2007). More recently, the same group reported a dosimetric analysis of 20 patients planned with a simplified intensity modulated radiation therapy (IMRT) to treat the breast in prone position. Dose

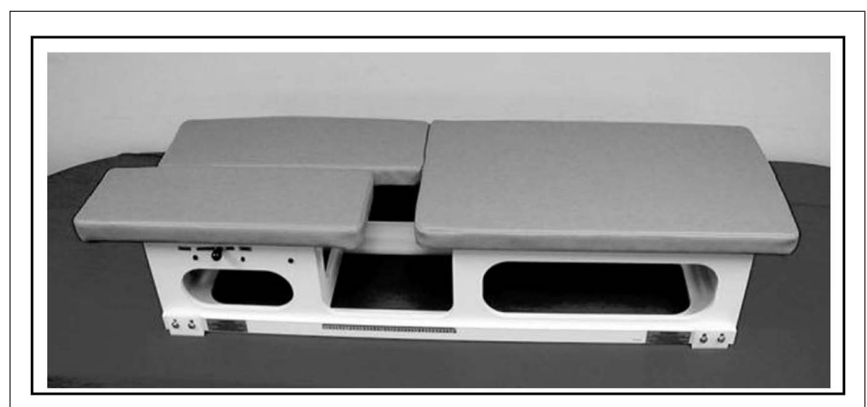

FIGURE 1 | Prone breast positioning table Victoreen ${ }^{\circledR}$ Model 37-018. The table was designed based on MSKCC experience. homogeneity was improved, particularly in women with larger, pendulous breasts (Goodman et al., 2004).

In addition, in 2009 a retrospective analysis of the outcome of 128 patients treated in the prone position, who had received the accelerated Canadian fractionation regimen $(42.4 \mathrm{~Gy}$ in 16 fractions of $2.65 \mathrm{~Gy}$ ) modified to include a sequential $10 \mathrm{~Gy}$ boost delivered in $2 \mathrm{~Gy}$ fractions was reported. At a median followup of 18 months, comparable early tumor control and cosmesis to those achieved by standard fractionation were demonstrated (Croog et al., 2009).

\section{THE USC EXPERIENCE OF PRONE BREAST RADIATION THERAPY}

In the early 1990s, we started exploring accelerated partial breast radiation in the prone position through a series of studies conducted at the University of Southern California (Luxton et al., 1991; Formenti et al., 2002). A radiosurgery-like technique was investigated, based on the use of multiple non-coplanar beams. The patient was positioned with arms kept adjacent to the chest on a home-built wooden platform (Figure 2). The platform had a system of removable concentrical inserts at the level of the breast. Each insert consisted of several concentrical ring components to accommodate different size breasts. With the contralateral insert in place to close the opening, the target breast was centered in the ipsilateral opening, adjusted to the specific breast size by using the appropriate ring inserts. The distal part of the board extended from the end of the treatment couch, enabling full couch rotation to use superior-oblique and superior-inferior beams. To prevent metal distortions at planning CT, the board did not contain metal parts: the thickness of the board was chosen to prevent flexibility under the patient's weight. This approach was designed for partial breast treatments only. It was incompatible with inclusion of the whole breast tissue by tangential fields since part of the breast tissue was superior to the opening of the platform: with the entire breast volume as target the tangential fields would have encountered the ipsilateral arm (kept adjacent to the chest) and the side bar (approximately two inches of wood). A beam eye view from below the platform easily demonstrated inclusion of only part of

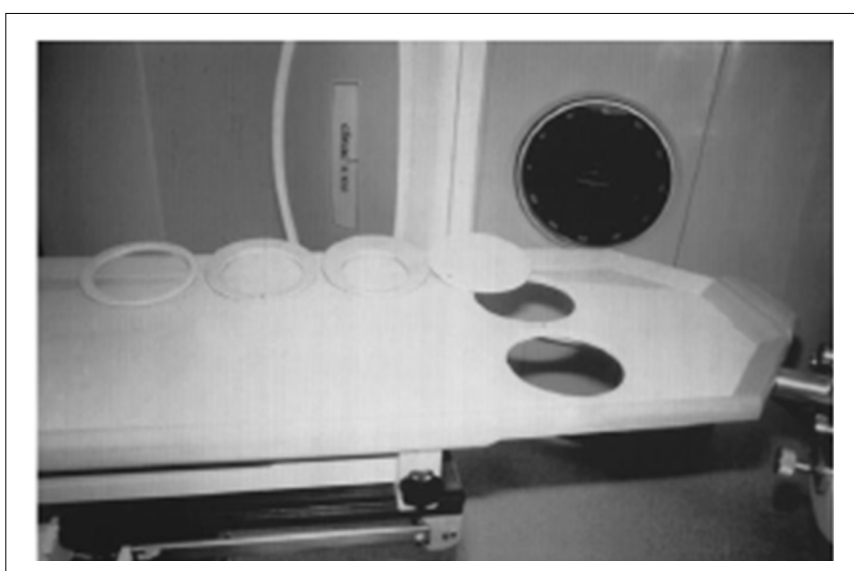

FIGURE 2 | USC prone breast board for partial breast treatment. The contralateral opening is covered by the solid disk, the indexed breast is hanging through one of the rings inserted into the ipsilateral opening. 
the breast tissue, a few $\mathrm{cm}$ distal from the bony reference of the rib cage.

This first platform (Figure 2) was used in a pilot-feasibility trial of ten consecutive patients who were assigned a five fractions regimens. Nine were treated with the proposed technique, with planning target volume (PTV) defined on the basis of CT findings at the visualized tumor cavity plus a $1-2-\mathrm{cm}$ margin. A $4 \mathrm{mV}$ $\mathrm{X}$-ray beam was used to deliver the prescribed dose through multiple fixed fields that consistently avoided exiting in the lung or heart, utilizing multiple couch rotations. All patients received five fractions over 10 days (total dose range, 25-30 Gy): three were randomly assigned to receive 5.0 Gy per fraction; four, 5.5 Gy; and two, 6.0 Gy, respectively. Cosmesis was assessed by patients and physicians before treatment and at least 36 months after treatment. At this minimum follow-up (range, 36-53 months) all patients were alive and disease-free with good to excellent cosmesis (Formenti et al., 2002). This experience established feasibility of prone PBI and justified further investigation of this approach.

\section{THE NYU EXPERIENCE: PRONE BREAST RADIATION DEVICES}

Research in prone breast irradiation continued at New York University with focus on designing a device compatible with prone "whole breast" radiotherapy. It became clear that in order to assure interfraction reproducibility, a very precise immobilization technique needed to be in place at the time of CT simulation to assure exact replication on the treatment table. Moreover, a CT simulator with a larger bore became necessary for imaging the patient on the prone table. A new breast board was built, eliminating the side bar characteristic of the USC design. In addition, the patient setup was changed to an "arms-up" position (Figure 3, version 1). Again, no metal was included in the board to prevent image distortion at CT planning. Since it was found that utilizing small tangential fields resulted in acceptable dose distribution, the requirement for full couch rotation became unnecessary, and permitted modification of the design of the table to accommodate a more comfortable position (Figure 3, version 2), which greatly increased patients' comfort. Made of solid wood, this version of the prone board was non-deformable with weight and relatively heavy, requiring two therapists to position it on the couch.

An additional step to ease transport of the board while at the same time enhancing the comfort of the prone patient was the introduction of the prone mattress, i.e., a modular breast board made of a thick memory foam layer placed on top of $12.5 \mathrm{~cm}$ Styrofoam $^{\mathrm{TM}}$ (Figure 3, version 3). The memory foam assures consistently reproducing the same degree of compression with weight, making the position reproducible for each patient over multiple radiation therapy sessions. Importantly, the prone mattress is light and easily removed from the couch. Its simple design has made it affordable and easy to build at multiple international radiation oncology centers. Limitations are the absence of a holding bar to maintain the arms and hands in the same position, and the fact that it was not designed to be specifically indexed to the couch, thus requiring therapists experienced in prone breast radiation and the accurate use of positioning lasers to prevent differences in a dayto-day setup (Device for prone breast radiotherapy. US Patent No. 7.763.864 B2).
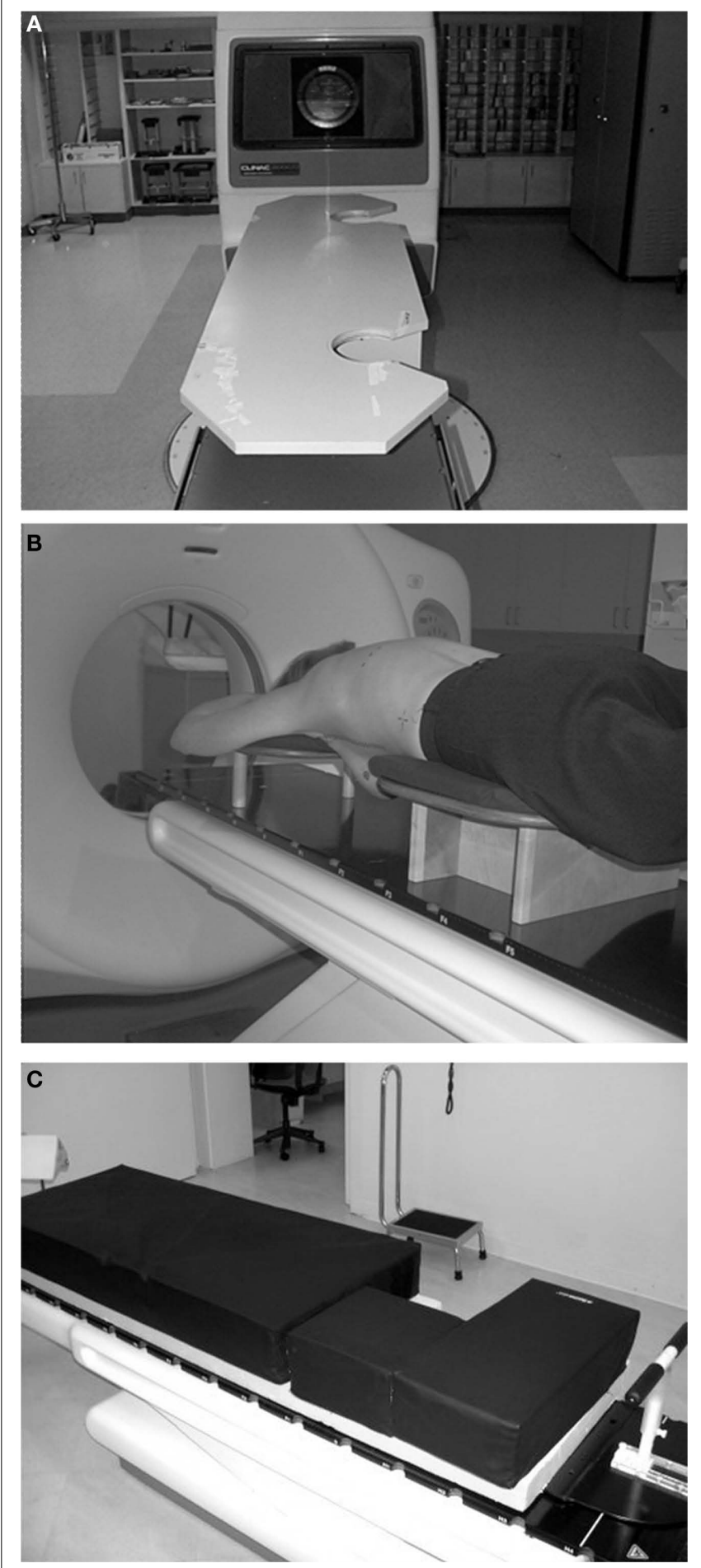

FIGURE 3 | (A) NYU design, version 1. The board extends over the treatment couch, allowing couch rotation. By eliminating the ipsilateral bars tangential fields are possible. (B) NYU design, version 2. The board fully rests on the treatment couch. The leg support allowed a thinner board; therefore a soft cushion could be placed on the top, for patient comfort. Couch rotation is limited $\left(<10^{\circ}\right)$. (C) NYU design, version 3. Two-inch thick memory foam layer on top of 5 inch Styrofoam ${ }^{\mathrm{TM}}$, wrapped in washable vinyl cover. (For large breast carriers, additional Styrofoam ${ }^{\mathrm{TM}}$ layers can be used under the mattress). 
The experience acquired in the design of each generation of devices was later shared in collaborations with industry. We provided un-remunerated feedback to the design of the commercial prone breast positioning devices of Orbital Therapy Inc. (ClearVue ${ }^{\mathrm{TM}}$ ) and Varian Medical Systems Inc. (Access $360^{\mathrm{TM}}$ ). The ClearVue ${ }^{\mathrm{TM}}$ model (Figure 4A,B) is a table-top design with good access and visibility for accurate setup. The soft cushion is ergonomically designed for greater patient comfort. It is built of carbon fiber, making it lightweight and causing very low radiation absorption. The Access $360^{\mathrm{TM}}$ model from Varian (Figure 5) currently undergoing evaluation is essentially an extension of the treatment couch. Built of carbon fiber, it has an additional opening of the soft cushioning lining to visualize the field more cranially if the supraclavicular region is a target. Although couch rotation is possible, large-angle superior-oblique fields are not feasible, as they would encounter the arms of the patient.

While better engineered devices have permitted better indexing of the table and more comfortable and reproducible positioning, the basic dosimetric advantages of a prone setup became apparent since the beginning, reflecting the more advantageous access to the target when gravity distances the breast parenchyma from the chest wall.

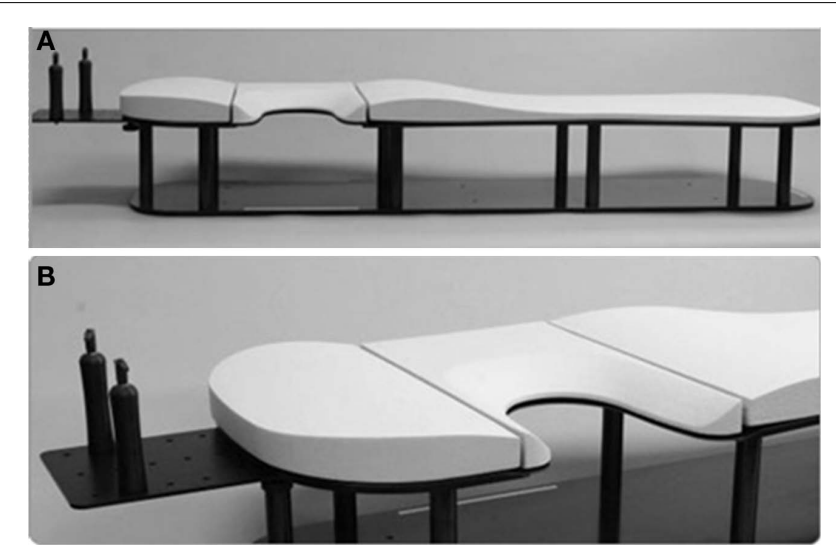

FIGURE 4 | (A) ClearVue ${ }^{\text {TM }}$ System (Orbital Therapy): carbon fiber plate and board, soft cover for patient comfort. (B) The central segment can be rotated for right breast patients.

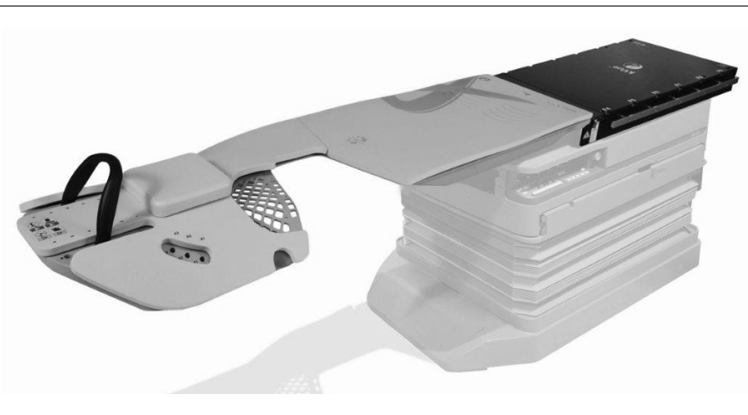

FIGURE 5 | Access $360^{\mathrm{TM}}$ (Varian Medical Systems): Built of carbon fiber mesh, with soft cushion on top. Attaches to the end of the treatment couch (replaces the upper section). An uncovered section of the mesh enables supraclavicular fields.

\section{RESULTS OF NYU PRONE BREAST RADIATION THERAPY TRIALS}

While many breast cancer patients requiring whole breast radiation therapy have been treated in the prone position with standard fractionation regimens, NYU conducted a series of clinical trials to test hypo-fractionated, accelerated regimens of breast radiotherapy.

The first NYU trial of prone radiation tested partial breast accelerated fractionation (PBI) as part of a DOD IDEA Grant "Hypo-Fractionated Conformal Radiation Therapy to the Tumor Bed after Segmental Mastectomy" awarded in 2000. Clinical and dose-volume histogram results of the first 47 patients accrued to this protocol were reported in 2004 (Formenti et al., 2004) and the 5-year results were recently presented at ASTRO (Formenti, 2010). All patients were treated prone with three-dimensional conformal radiotherapy after breast conserving surgery. Eligibility criteria were postmenopausal women with Stage T1N0 breast cancer that had initially refused to undergo 6 weeks of standard radiotherapy and were interested in a shorter regimen. The postoperative cavity was defined as the clinical target volume with a $1.5-\mathrm{cm}$ margin added to determine the PTV. The prescription dose was $30 \mathrm{~Gy}$ at $6 \mathrm{~Gy} /$ fraction in five fractions on Monday, Wednesday, Friday, Monday, and Wednesday.

The encouraging experience with prone PBI provided the foundations to test prone radiotherapy targeting the whole index breast. In 2002, NYU started a Phase I-II trial of prone accelerated intensity modulated radiation therapy to the whole breast that included a concomitant boost to the tumor bed (Dewyngaert et al., 2007). This approach was chosen to open the access to hypo-fractionation to patients with more aggressive tumors with potentially higher risk of local recurrence. Eligible patients for this IRB-approved prospective trial were stage I or II who had undergone breast conserving surgery with negative margins. A dose of $40.5 \mathrm{~Gy}$, delivered at $2.7 \mathrm{~Gy}$ in 15 fractions, was prescribed to the index breast with an additional concomitant boost of $0.5 \mathrm{~Gy}$ delivered to the tumor bed, for a total dose of $48 \mathrm{~Gy}$ to the lumpectomy site. Dosimetric results of the trial were reported in 2007 after 91 patients had been treated, with a median follow-up of 12 months (Formenti et al., 2007). The technique was feasible in all patients regardless of breast volume. Sparing of the heart was achieved as prescribed by the protocol, that required limiting $5 \%$ of the heart volume to receive $\geq 18$ Gy and $\leq 10 \%$ of the ipsilateral lung volume to receive $\geq 20$ Gy.

\section{REPRODUCIBILITY OF THE NYU PRONE TECHNIQUE}

The advantages of a prone setup have long been recognized in diagnostic radiology where both breast biopsies and breast MRI are routinely performed in the prone position since it minimizes the motility of the anterior chest wall, reducing movement artifact (Wolfman et al., 1985).

Over the years, we have developed a reliable system to reproduce for treatment the position imaged at simulation. At the time of simulation with the patient lying in the supine position, the superior, inferior, and mid-axillary borders were marked with fiducials and the patient's midline is defined by the placement of a radio-opaque wire over the sternum. Afterward, the patient is positioned prone with the breast situated approximately in the 
center of the opening of the breast board. The plane passing through the nipple is defined by laser lights and it is used as a reference plane to establish triangulation tattoos for leveling the patient on a daily basis. Three tattoos define this plane: two lateral leveling tattoos at mid-level of the torso in the anterior-posterior dimension and a posterior tattoo, on the back of the patient, offmidline in the direction of the breast side that is being treated. The latter provides the therapist with a final check to confirm, once prone, whether the right or left breast is treated (Figure 6A,B). An additional tattoo is placed on the skin of the treated breast to locate the isocenter prior to each treatment. The breast tattoo indicates the superior-inferior and anterior-posterior level of the isocenter. In this way, the isocenter is defined simply by moving along a horizontal line passing through the breast tattoo. The depth of the isocenter is chosen close to midline, maintaining a minimum depth of $1.5 \mathrm{~cm}$ in tissue on the medial aspect of the breast. Applying this method of spatial reference, NYU has reported the interfraction and intrafraction setup variability for prone breast radiation therapy in a series of consecutive patients enrolled in the concomitant boost protocol (Mitchell et al., 2010). An electronic portal device with cine capability acquired images on 10 consecutive patients: inter- and intra-fraction variability
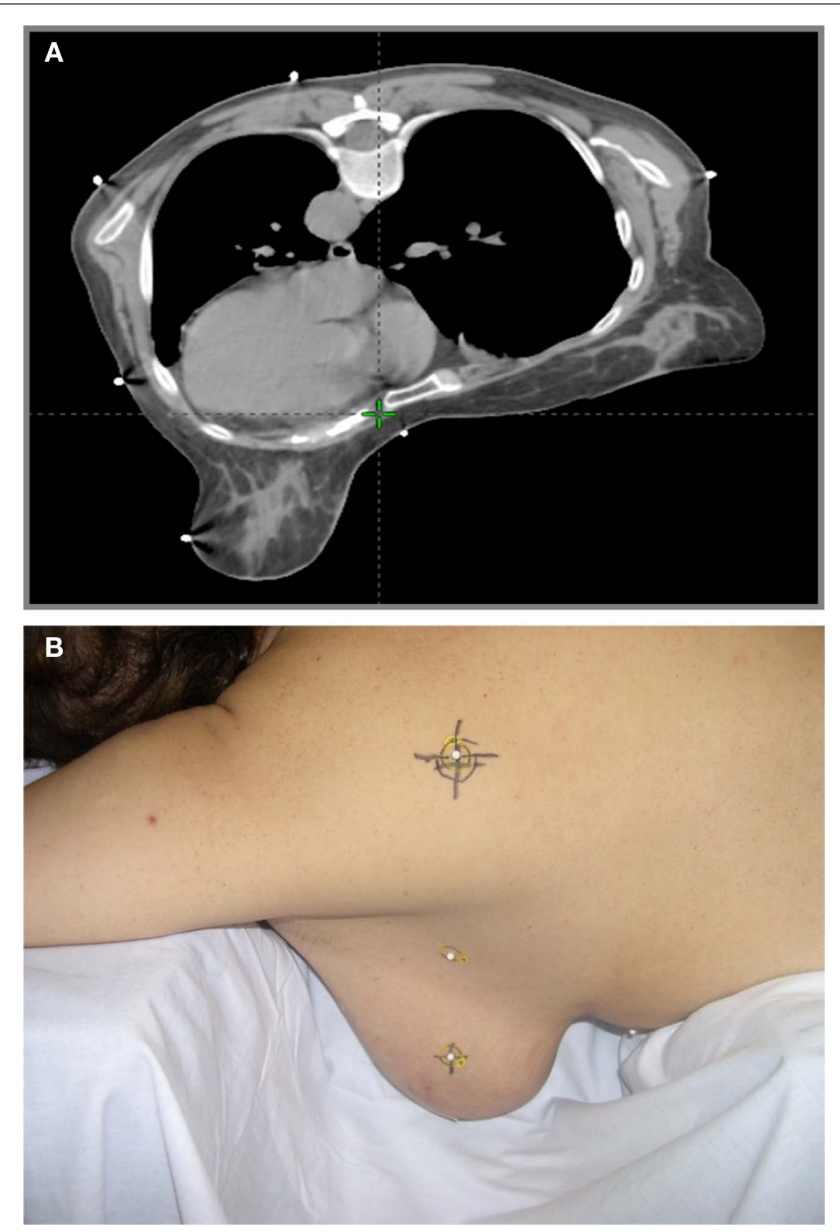

FIGURE 6 | (A,B) Placement of the localization and setup BBs. of the fiducial markers were demonstrated to range from 0.02 to $0.14 \mathrm{~cm}$. The findings resulted in a CTV to PTV expansion of $1.4 \mathrm{~cm}$, confirming the adequacy of our current protocol of $1.5 \mathrm{~cm}$ expansion.

In another study, the role of cone beam computerized tomography (CBCT) imaging to assure accurate/reproducible partial breast setup was analyzed in $343 \mathrm{CBCT}$, from 70 consecutive patients (Jozsef et al., 2011). The patient was positioned utilizing lasers and markers/tattoos, then portal images were taken, but no adjustment implemented. This was followed by CBCT and the suggested shifts were applied. A second set of portal images then verified the corrected setup. If the images did not accurately reproduce the digital radiograph generated after simulation, the patient was repositioned and the procedure was repeated. The analysis of the shifts detected led to the following results:

(a) In $7 / 343$ cases (2\%) was a repeated setup necessary.

(b) The average shifts over five fractions never exceeded $1.3 \mathrm{~cm}$ (in any direction and in any patients).

(c) The average magnitude of shifts (sum of squares of the shifts in the three principal directions) was $0.68 \mathrm{~cm}$.

(d) In three patients $(4.3 \%)$ was a shift exceeding $1.5 \mathrm{~cm}$ in any direction detected more than once during the course.

We concluded that the setup is reasonably accurate and reproducible, and that a $1.5-\mathrm{cm}$ margin is sufficient. This margin size was dosimetrically verified by modeling the summed dose distribution over five fractions for the first 21 patients. Specifically, $95 \%$ of the CTV (surgical cavity plus $2-3 \mathrm{~mm}$ ) was covered by the prescription dose in all but one case $(20 / 21,95.2 \%)$. Furthermore, $95 \%$ of the prescription dose covered $95 \%$ of the PTV-Eval (CTV plus $1.5 \mathrm{~cm}$ margin, excluding possible overlapping lung and $0.7 \mathrm{~cm}$ thick superficial layer from the skin) in $19 / 21$ patients (90.5\%; Jozsef et al., 2011).

Other groups have compared prone versus supine treatment utilizing 4D-CT, demonstrating decreased intrafraction variation from respiration. Morrow et al. (2007) demonstrated that respiratory motion of the chest wall was drastically reduced from $2.3 \pm 0.9 \mathrm{~mm}$ in supine position to $-0.1 \pm 0.4 \mathrm{~mm}$ in prone position utilizing 4D-CT in three patients. Kirby et al. (2010) had similar results in 25 patients. In contrast to our experience, both groups found greater systematic and random setup errors with the prone technique as compared to supine treatment. Neither group, however, utilized additional fiducial marks as we do to better reproduce the daily setup.

\section{HEART EXPOSURE WHEN PRONE}

A concern raised regarding prone breast irradiation is the displacement of the heart anteriorly when prone. Increased mortality from heart disease 10-15 years after receiving irradiation to the left breast compared to patients irradiated to the right breast demonstrated the late cardiovascular morbidity associated with inclusion of the organ in the radiation field. Consistently, the volume of heart in the field correlates with perfusion deficits and microvascular disease and inclusion in the field of the left anterior descending artery contributes to atherosclerosis and coronary artery disease (Darby et al., 2010). 
Investigators at Duke University retrospectively compared simulation CT scans of 16 patients treated in the supine position with breast MRI's obtained in the prone position (Chino and Marks, 2008). The mean displacement of the heart was $19 \mathrm{~mm}$ anteriorly to study whether the prone setup would better spare the heart. Concerns about this work have been discussed (Lymberis and Formenti, 2008).

However, we also occasionally noticed a similar positional shift of the heart early in the beginning of the NYU experience with prone breast irradiation, attributable to a "sinking" of the left chest wall and breast tissue below the board with an axial rotation toward the opening of the board. Rarely in patients when the heart moves by gravity in the same direction does it reach the chest wall below the level of the prone board, and is encompassed by the tangent treatment fields. By turning the patient's head toward the treated breast we have corrected for this rotation and drastically limited this problem. With this correction, during the past 400 prone simulations for left breast cancer, none of the patients were found to have any volume of heart included in the tangent fields.

To better study this issue and that of possible anatomic variance among patients, we designed NYU Trial 05-181 in which 400 patients, 200 with left and 200 with right breast cancer, consented to undergo two CT simulations for planning both prone and supine (Formenti et al., 2009).

The results of this trial demonstrated that in $15 \%$ of patients with left breast target lesions, the supine position decreased the amount of heart in the field by a mean of $6 \mathrm{cc}$. Eighty-five percent of the patients with left breast target lesions benefited by the prone treatment, decreasing the amount of heart in the field by a mean of $11 \mathrm{cc}$. Sixty-nine percent of the left-sided breast patients with an A/B cup ( $<750 \mathrm{cc}$ breast volume) benefited from treatment in the prone position, as did $96 \%$ with a $C$ cup $(750-1500 \mathrm{cc})$ and $99 \%$ with a D cup $(>1500 \mathrm{cc})$. These results have modified our standard practice of breast radiation therapy: we now simulate all patients in prone position and treat prone unless part of the heart is included in the tangent field. In the latter case, the patients are re-simulated supine.

\section{COMMON CHALLENGES OF A PRONE SETUP}

By having treated several thousands of women over the past 15 years, we have accumulated an extensive experience on how to optimally set up a patient in the prone position, and have identified many of the challenges with this setup. Twice a year, NYU offers a 2-days Continuous Medical Education course, to share our experience in prone breast radiotherapy. Through the interaction with the participants of these sessions, we have identified several common areas of confusion or error. These include: (1) inadequate coverage of the target, i.e., the entire parenchyma of the index breast; (2) incorrect positioning of the patient, particularly in women with large breast size; (3) allowing for excessive "sinking" and rotation of the patient toward the opening of the board.

\section{ASSURING WHOLE BREAST COVERAGE}

The first and most important issue in whole breast prone radiotherapy, is the recognition of the boundaries of the index breast when prone. At the time of simulation radio-opaque be-bees are paced on the reference plane, to define the superior, inferior, and medial edges of the target. Specifically, the superior edge of the field is defined at the inferior margin of the clavicular head. The inferior margin is placed at $2 \mathrm{~cm}$ below infra-mammary fold, and the medial margin on the midline of the patient, anterior to the sternum. The posterior edge is defined by CT imaging through a virtual line that connects on the reference transverse plane the midline, sternal marker to a point on the skin of the lateral chest wall which is just anterior to the edge of the latissimus dorsi muscle, to assures inclusion of the breast parenchyma. While the superior, medial, and inferior margins can be easily maintained consistent with those of a supine setup the lateral and deep margins are often a source of confusion.

Part of the confusion reflects the fact that, when prone the pectoralis major may also move away from the chest wall by gravity: since the breast tissue is external to the muscle, the virtual line that defines the deeper edge of the tangent fields, lies almost always external to the ribcage.

\section{PREVENTING INCORRECT POSITIONING}

A frequently observed mistake consists of inadequate alignment of the patient on the table at the time of prone CT simulation. Figure 7 demonstrates a typical case: in panel G1 the patient is initially set up with the sternum lateral to the edge of the mattress. In this case, the contralateral breast creates a wedge at the

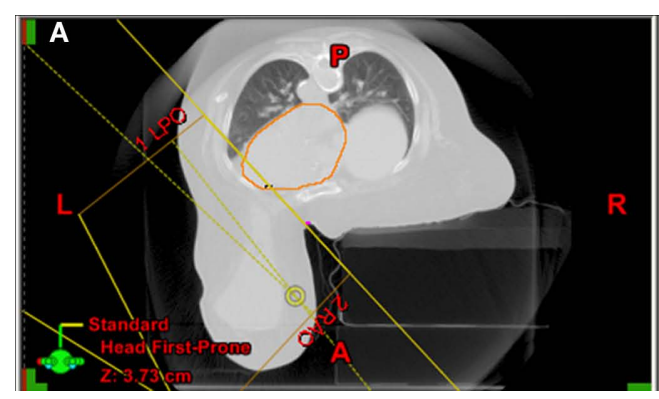

FIGURE 7 | (A) Initial set up with the sternum lateral to the edge of the mattress and the contralateral breast creating a wedge at the midline. To avoid the contralateral breast the angle of the tangents would include part of the heart and LAD. (B) Once correctly aligned with the sternum more medial

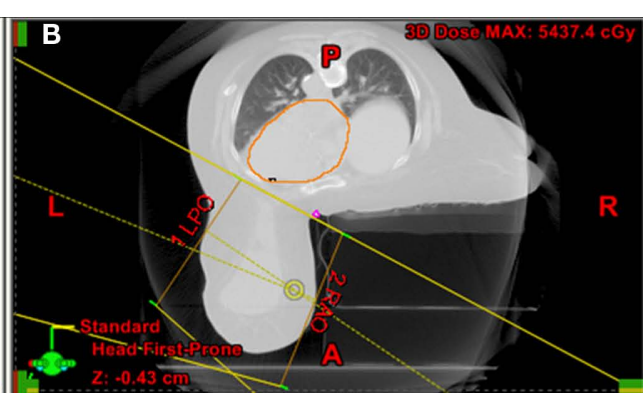

toward the edge of the mattress and the contralateral breast better displaced, the angle of the tangents permits exclusion of the contralateral breast, heart, and $L A D$, while including the entire parenchyma of the index breast (lateral edge is just anterior to the latissimus dorsi). 
midline. To avoid inclusion in the field of the contralateral breast the angle of the tangents would include part of the heart and LAD. This patient required re-simulation: once correctly aligned with the sternum more medial toward the edge of the mattress and the contralateral breast better displaced, the tangents avoided the contralateral breast and the heart and LAD, while including the entire parenchyma of the index breast (lateral edge is just anterior to the latissimus dorsi).

\section{PREVENTING "SINKING" AND ROTATION OF THE PATIENT TOWARD THE OPENING OF THE BOARD}

As mentioned before, this issue was prevalent at the beginning of our experience. Two interventions corrected this problem. First, we try to position the sternum of the patient on the edge of the board, and assuring support of the board at the midline. Second, turning the patient's head laterally toward the treated breast, consistently prevents this effect. The rotation of the neck, physiologically reflects in an adjustment of the spine and the rest of the body that compensates for "sinking" and axial rotation toward the index breast. With these two precautions, during the past 463 prone simulations for left breast cancer, none of the patients were found to have any volume of heart included in the tangent fields.

\section{LIMITATIONS OF THE PRONE TECHNIQUE AND CURRENT NYU RESEARCH}

The experience with a prone setup for breast radiotherapy has identified some of the limitations of this approach and has inspired a series of ongoing trials to address new scientific questions.

As stated earlier, we routinely use a prone simulation for both trial and standard fractionation regimens. Neither breast size nor patient's weight have ever presented a problem. Exceptions include patients requiring four-fields radiation therapy to include the axillary nodes and patients in our trials of concurrent chemoradiation for locally advanced breast cancer (Adams et al., 2010) and those rare patients who cannot tolerate the prone decubitus. A common complaint is pain from the tension on the neck and spine muscles, to maintain the position. Some of the commercially available prone boards include an head-holder similar to those used for body massage, that enables support with a straight neck. We reserve this approach to women with history of neck injury or disk problems.

Another identified limitation of the prone technique is a decrease in axillary nodal coverage (Alonso-Basanta et al., 2009). Dosimetry of the axilla (level I-III nodes) was assessed in twenty patients imaged and planned both supine and prone. Standard tangent fields were designed for the whole breast to deliver a

\section{REFERENCES}

Adams, S., Chakravarthy, A. B., Donach, M., Spicer, D., Lymberis, S., Singh, B., Bauer, J. A., Hochman, T., Goldberg, J. D., Muggia, F., Schneider, R. J., Pietenpol, J. A., and Formenti, S. C. (2010). Preoperative concurrent paclitaxel-radiation in locally advanced breast cancer: pathologic response correlates with five-year overall survival. Breast Cancer Res. Treat. 124, 723-732.
Alonso-Basanta, M., Ko, J., Babcock, M., Dewyngaert, J. K., and Formenti, S. C. (2009). Coverage of axillary lymph nodes in supine vs. prone breast radiotherapy. Int. J. Radiat. Oncol. Biol. Phys. 73, 745-751.

Baclesse, G., Gricouroff, G., and Taillefer, A. (1939). Essai de Roentgentherapie du cancer du sein suive d'operation large. Resultats histologiques. Bull. Cancer 28, 729-743.

prescribed dose of $50 \mathrm{~Gy}$. Dose-volume histograms were compared between the two sets. For each patient, coverage of breast tissue and tumor bed was readily achieved by either technique. However, in either position; treatment of the nodal regions was inadequate, and, on average, the mean dose to levels I-III axillary nodes was approximately $50 \%$ less in the prone as compared to the supine position. A prone setup is generally inadequate in patients requiring radiotherapy to axillary nodes. However, a prone technique that targets the breast and level 3 and supraclavicular nodes is feasible (Sethi et al., 2011) and it is being tested in a prospective trial of breast cancer patients with $<5$ involved axillary nodes, after an adequate axillary dissection.

Similarly, we have questioned the need of IMRT when treating prone (Hardee et al., 2011): we are currently conducting a prospective randomized study that tests the role of a weekly boost, instead of a daily concomitant boost. If proven equivalent, a weekly boost could enable prone breast without IMRT, making this approach more feasible and cost-effective.

Finally, the combination of concurrent carboplatin and accelerated adjuvant radiotherapy is explored in a trial open to triple negative tumor carriers and the role of imaged guided radiotherapy in studied as part of PBI.

\section{CONCLUSION}

Over the years, our team has developed considerable experience in prone radiotherapy of the breast. In the interest of space, many details were omitted and this review focuses on the most relevant issues on this subject. Reproducibility of the daily setup is of tantamount importance in being able to exploit the advantage of immobilization of the chest wall and breast by reducing breathing motion when prone, and it is the very first step to assure the success of this approach. Importantly, a prone setup is advantageous to most women needing breast radiation therapy, independently from their breast size. When correctly positioned prone, it is possible to exclude the heart and drastically decrease the amount of lung in the field compared to supine, preventing late effects to the normal tissue. Moreover, the decreased acute toxicities of this approach, despite the inclusion of a concomitant boost to the tumor bed in accelerated, shorter radiotherapy regimens, have made it desirable to many patients.

However, prone accelerated breast radiotherapy with a concomitant boost remains a research approach. Its equivalence to a standard fractionation regimen is under investigation in RTOG 1005: the results of this prospective trial will decide if it can become a standard choice in breast radiotherapy.

Bentel, G. C., and Marks, L. B. (1994). A simple device to position large/flaccid breasts during tangential breast irradiation. Int J. Radiat. Oncol. Biol. Phys. 29, 879-882.

Bentel, G. C., Marks, L. B., Whiddon, C. S., and Prosnitz, L. R. (1999). Acute and late morbidity of using a breast positioning ring in women with large/pendulous breasts. Radiother. Oncol. 50, 277-281.
Chino, J. P., and Marks, L. B. (2008). Prone positioning causes the heart to be displaced anteriorly within the thorax: implications for breast cancer treatment. Int. J. Radiat. Oncol. Biol. Phys. 70, 916-920.

Croog, V. J., Wu, A. J., Mccormick, B., and Beal, K. P. (2009). Accelerated whole breast irradiation with intensity-modulated radiotherapy to the prone breast. Int. J. Radiat. Oncol. Biol. Phys. 73, 88-93. 
Cross, M. A., Elson, H. R., and Aron, B. S. (1989). Breast conservation radiation therapy technique for women with large breasts. Int. J. Radiat. Oncol. Biol. Phys. 17, 199-203.

Darby, S. C., Cutter, D. J., Boerma, M., Constine, L. S., Fajardo, L. F., Kodama, K., Mabuchi, K., Marks, L. B., Mettler, F. A., Pierce, L. J., Trott, K. R., Yeh, E. T., and Shore, R. E. (2010). Radiation-related heart disease: current knowledge and future prospects. Int. J. Radiat. Oncol. Biol. Phys. 76, 656-665.

Dewyngaert, J. K., Jozsef, G., Mitchell, J., Rosenstein, B., and Formenti, S. C. (2007). Accelerated intensitymodulated radiotherapy to breast in prone position: dosimetric results. Int. J. Radiat. Oncol. Biol. Phys. 68, 1251-1259.

Fisher, B., Anderson, S., Bryant, J., Margolese, R. G., Deutsch, M., Fisher, E. R., Jeong, J., and Wolmark, N. (2002). Twenty-year follow-up of a randomized trial comparing total mastectomy, lumpectomy, and lumpectomy plus irradiation for the treatment of invasive breast cancer. (National Surgical Adjuvant Breast and Bowel Project, Pittsburg, PA). N. Engl. J. Med. 347, 1233-1241.

Formenti, S. (2010). Five-year results of prone accelerated partial breast irradiation: NYU 00-23. Int. J. Radiat. Oncol. Biol. Phys. 78(Suppl.), Abstract No. 2004.

Formenti, S. C., Gidea-Addeo, D., Goldberg, J. D., Roses, D. F., Guth, A., Rosenstein, B. S., and Dewyngaert, K. J. (2007). Phase I-II trial of prone accelerated intensity modulated radiation therapy to the breast to optimally spare normal tissue. $J$. Clin. Oncol. 25, 2236-2242.

Formenti, S. C., Lymberis, S., Parhar, P., Fenton-Kerimian, C., Magnolfi, B., Wen, B., Chang, J., and Dewyngaert, J. (2009). Results of NYU 05-181: a prospective trial to determine optimal position (prone versus supine) for breast radiotherapy. Paper Presented at the Proceedings of the American Society for Radiation Oncology 51st Annual Meeting, Chicago, IL, USA.

Formenti, S. C., Rosenstein, B., Skinner, K. A., and Jozsef, G. (2002). $\mathrm{T} 1$ stage breast cancer: adjuvant hypofractionated conformal radiation therapy to tumor bed in selected postmenopausal breast cancer patients - pilot feasibility study. Radiology 222, 171-178.

Formenti, S. C., Truong, M. T., Goldberg, J. D., Mukhi, V., Rosenstein, B., Roses, D., Shapiro, R., Guth, A., and Dewyngaert, J. K. (2004). Prone accelerated partial breast irradiation after breast-conserving surgery: preliminary clinical results and dose-volume histogram analysis. Int. J. Radiat. Oncol. Biol. Phys. 60, 493-504.

Fourquet, A., Campana, F., Rosenwald, J. C., and Vilcoq, J. R. (1991) Breast irradiation in the lateral decubitus position: technique of the Institut Curie. Radiother. Oncol. 22, 261-265.

Goodman, K. A., Hong, L., Wagman, R., Hunt, M. A., and Mccormick, B. (2004). Dosimetric analysis of a simplified intensity modulation technique for prone breast radiotherapy. Int. J. Radiat. Oncol. Biol. Phys. 60, 95-102.

Grann, A., Mccormick, B., Chabner, E. S., Gollamudi, S. V., Schupak, K. D., Mychalczak, B. R., Heerdt, A. S., Merchant, T. E., and Hunt, M. A. (2000). Prone breast radiotherapy in earlystage breast cancer: a preliminary analysis. Int. J. Radiat. Oncol. Biol. Phys. 47, 319-325.

Gray, J. R., Mccormick, B., Cox, L., and Yahalom, J. (1991). Primary breast irradiation in large-breasted or heavy women: analysis of cosmetic outcome. Int. J. Radiat. Oncol. Biol. Phys. 21, 347-354.

Hardee, M. E., Raza, S., Becker, S. J., Jozsef, G., Lymberis, S. C., Hochman, T., Goldberg, J. D., DeWyngaert, K. J., and Formenti, S. C. (2011). Prone hypofractionated whole-breast radiotherapy without a boost to the tumor bed: Compararable toxicity of IMRT versus a $3 \mathrm{D}$ conformal technique. Int. J. Radiat. Oncol. Biol. Phys. (in press).

Jelden, G. L., Chernak, E. S., RodriguezAntunez, A., Haaga, J. R., Lavik, P. S., and Dhaliwal, R. S. (1976). Further progress in CT scanning and computerized radiation therapy treatment planning. AJR Am. J. Roentgenol. 127, 179-185.

Jozsef, G., Dewyngaert, J. K., Becker, S. J., Lymberis, S. C., and Formenti, S.
C. (2011). Prospective study of conebeam computed tomography imageguided radiotherapy for prone accelerated partial breast irradiation. Int. J. Radiat. Oncol. Biol. Phys. 81, 568-574.

Kirby, A. M., Evans, P. M., Donovan, E. M., Convery, H. M., Haviland, J. S., and Yarnold, J. R. (2010). Prone versus supine positioning for whole and partial-breast radiotherapy: a comparison of non-target tissue dosimetry. Radiother. Oncol. 96, 178-184.

Luxton, G., Jozsef, G., and Astrahan, M. A. (1991). Algorithm for dosimetry of multiarc linear-accelerator stereotactic radiosurgery. Med. Phys. 18, 1211-1221.

Lymberis, S. C., and Formenti, S. C. (2008). Prone-breast radiotherapy: too early for conclusions: in regard to Chino et $\mathrm{Al}$. (Int $\mathrm{j}$ radiat oncol biol phys 2008; 70:916-920). Int. J. Radiat. Oncol. Biol. Phys. 72, 301-302; author reply 302.

Merchant, T. E., and Mccormick, B. (1994). Prone position breast irradiation. Int. J. Radiat. Oncol. Biol. Phys. 30, 197-203.

Mitchell, J., Formenti, S. C., and Dewyngaert, J. K. (2010). Interfraction and intrafraction setup variability for prone breast radiation therapy. Int. J. Radiat. Oncol. Biol. Phys. 76, 1571-1577.

Monson, J. M., Chin, L., Nixon, A., Gage, I., Silver, B., Recht, A., and Harris, J. R. (1997). Is machine energy (4-8 MV) associated with outcome for stage I-II breast cancer patients? Int. J. Radiat. Oncol. Biol. Phys. 37 , 1095-1100.

Morrow, N. V., Stepaniak, C., White, J., Wilson, J. F., and Li, X. A. (2007). Intra- and interfractional variations for prone breast irradiation: an indication for image-guided radiotherapy. Int. J. Radiat. Oncol. Biol. Phys. 69, 910-917.

Sethi, R., No, H. S., Jozsef, G., Ko, J. P., and Formenti, S. C. (2011). Comparison of three-dimensional versus intensity-modulated radiotherapy techniques to treat breast and axillary level III and supraclavicular nodes in a prone versus supine position. Radiother. Oncol. (in press).

Solin, L. J., Chu, J. C., Sontag, M. R., Brewster, L., Cheng, E., Doppke, K., Drzymala, R. E., Hunt, M.,
Kuske, R., and Manolis, J. M. (1991). Three-dimensional photon treatment planning of the intact breast. Int. J. Radiat. Oncol. Biol. Phys. 21, 193-203.

Stegman, L. D., Beal, K. P., Hunt, M. A., Fornier, M. N., and Mccormick, B. (2007). Long-term clinical outcomes of whole-breast irradiation delivered in the prone position. Int J. Radiat. Oncol. Biol. Phys. 68, 73-81.

Veronesi, U., Cascinelli, N., Mariani, L., Greco, M., Saccozzi, R. Luini, A., Aguilar, M., and Marubini, E. (2002). Twenty-year followup of a randomized study comparing breast-conserving surgery with radical mastectomy for early breast cancer. N. Engl. J. Med. 347, 1227-1232.

Wolfman, N. T., Moran, R., Moran, P. R., and Karstaedt, N. (1985). Simultaneous MR imaging of both breasts using a dedicated receiver coil. Radiology 155, 241-243.

Zierhut, D., Flentje, M., Frank, C., Oetzel, D., and Wannenmacher, M. (1994). Conservative treatment of breast cancer: modified irradiation technique for women with large breasts. Radiother. Oncol. 31, 256-261.

Conflict of Interest Statement: The authors declare that the research was conducted in the absence of any commercial or financial relationships that could be construed as a potential conflict of interest.

Received: 12 August 2011; accepted: 20 September 2011; published online: 11 October 2011.

Citation: Huppert N, Jozsef G, DeWyngaert $K$ and Formenti SC (2011) The role of a prone setup in breast radiation therapy. Front. Oncol. 1:31. doi: 10.3389/fonc.2011.00031

This article was submitted to Frontiers in Radiation Oncology, a specialty of Frontiers in Oncology.

Copyright (c) 2011 Huppert, Jozsef, DeWyngaert and Formenti. This is an open-access article subject to a nonexclusive license between the authors and Frontiers Media SA, which permits use, distribution and reproduction in other forums, provided the original authors and source are credited and other Frontiers conditions are complied with. 